\title{
PROSPECCIÓN ARQUEOLÓGICA EN AMÉRICA CENTRAL MEDIANTE EL USO DE MÉTODOS GEOFÍSICOS
}

\author{
Mario E. Arias ${ }^{1,3}$ \& Alexis Mojica ${ }^{2,3}$ \\ ${ }^{1}$ Escuela Centroamericana de Geología, Apdo. 2-14, 2060 Universidad de Costa Rica, \\ marioa@geologia.ucr.ac.cr \\ ${ }^{2}$ Instituto de Geociencias, CITEN. Apdo. 10971 Universidad de Panamá \\ amojica27@ netexplora.com \\ ${ }^{3}$ Département de Géophysique Appliquée, UMR 7619 Sisyphe, Université Pierre \\ et Marie Curie
}

(Recibido 19/8/03; aceptado 3/3/04)

\begin{abstract}
The geophysical methods of prospecting are considered as an important tool in the archaeological detection, it allows to detect the remains of structures buried, thanks to the study of some of their physical properties. The numeric analysis of the electric, magnetic, and electromagnetic signs, products of the application of the cartography techniques in two important archaeological place of the Central American region that are presented in this article, have allowed to put in evidence the contrast in the physical properties of the archaeological remains as well as of the adjacent floor.
\end{abstract}

Keywords: Archaeological prospecting, Geophysical methods, Central America.

RESUMEN: Los métodos geofísicos de prospección son considerados como una herramienta importante en la detección arqueológica ya que permite detectar y parametrizar los restos de estructuras enterradas gracias al estudio de algunas de las propiedades físicas del mismo. El análisis numérico de las señales eléctricas, magnéticas y electromagnéticas, productos de la aplicación de las técnicas de cartografía y perfilaje en dos sitios arqueológicamante importantes de la región centroamericana que se presentan en este artículo, ha permitido poner en evidencia el contraste en las propiedades físicas de los restos arqueológicos así como del suelo adyacente.

Palabras clave: Prospección arqueológica, Métodos geofísicos, América Central.

\section{INTRODUCCIÓN}

El estudio de un sitio arqueológico mediante el uso de técnicas geofísicas, es posible gracias a que los asentamientos humanos modifican el ciclo natural de la formación del suelo y producen alteraciones físicas. La intensidad de tales modificaciones depende del tiempo de ocupación, el número de habitantes y el tipo de actividades realizadas. 
Cuando se inicia una prospección geofísica, sea cual sea la índole de ésta, hay una serie de factores que influyen notablemente en la elección del método más apropiado. Como norma general no se suele emplear un solo método, sino dos o más que se complementen y hagan la investigación más eficiente. En todo caso, carece de sentido la aplicación de los métodos geofísicos si no se sigue una ordenada y cuidadosa campaña geofísica que debe programarse adecuadamente e incluir el trabajo en conjunto con arqueólogos, pedólogos e historiadores, para este caso específico.

La secuencia lógica de toda campaña geofísica consta de cinco etapas (Arias, 2000):

1. Planeamiento: se definir el problema que se quiere investigar; el papel protagónico lo debe tener el arqueólogo.

2. Planificación: traducir en términos geofísicos el problema planteado, elaborar el modelo del subsuelo cuya realidad quiere comprobarse.

3. Medición: corresponde con la toma de datos en el terreno minimizando los errores de muestreo.

4. Procesamiento: mediante un análisis numérico específico se tratan los datos.

5. Interpretación: el documento geofísico incorpora la expresión arqueológica, etapa en la cual, la interpretación de los resultados debe realizarse de una manera conjunta.

\section{LOS PRINCIPALES MÉTODOS GEOFÍSICOS UTILIZADOS EN LA ARQUEOLOGÍA}

Existe una gran variedad de métodos geofísicos de prospección, sin embargo, no todos son utilizados con fines arqueológicos. Se acostumbra diferenciar a aquellos métodos que solamente miden las variaciones de las propiedades físicas del subsuelo como métodos pasivos; por otra parte, cuando se produce una alteración del subsuelo y mide la respuesta, se consideran métodos activos.

\section{Prospección Geoeléctrica}

Los métodos geoeléctricos se basan en el estudio de la resistividad eléctrica, la cual revela el grado de oposición que determinado volumen de terreno ofrece al paso de las cargas eléctricas. Se emplean mecanismos basados en la inyección de corriente continua, utilizando un dispositivo de al menos cuatro electrodos que se sitúan sobre el terreno en contacto eléctrico con el medio rocoso a estudiar.

La medición de la resistividad eléctrica del subsuelo, corresponde con una técnica geofísica de tipo activa; que se basa en el análisis del contraste de las propiedades eléctricas del suelo. En este caso, el contraste de propiedades entre los restos arqueológicos y su contexto depende de la naturaleza de los materiales, la profundidad y forma de los restos, la proximidad entre ellos, grado de salinidad y la humedad del suelo. Si este contraste es suficientemente grande, las características arqueológicas podrán ser detectadas.

\section{Prospección magnética}

El reconocimiento magnético es la técnica de prospección más ampliamente usada en arqueología, debido a su confiabilidad y fácil uso. Se basa en la medición de pequeños cambios en las propiedades magnéticas del terreno y está considerada como una técnica geofísica pasiva. En 1958 cuando Aitken aplicó el magnetómetro de protones a la arqueología, el objetivo era detectar el magnetismo termoremanente producido por hornos y fuego, pero esos primeros experimentos mostraron nuevas posibilidades de aplicación para descubrir otros rasgos arqueológicos con menos diferencias en su susceptibilidad magnética.

En Arqueología, los magnetómetros más comunes son los protónicos, capaces de medir pequeñas variaciones en la intensidad del campo magnético total. Con este equipo es posible registrar lecturas en distintos puntos de un sitio arqueológico; el recorrido sistemático de la superficie 
permitirá la interpretación final. Actualmente podemos utilizar también el gradiómetro de vapor de Cesio, el cual permite obtener mayor información del sitio, debido a la frecuencia de medición y la mecánica de adquisición de datos. Gracias a estos dispositivos, es posible llevar a cabo la medición del gradiente magnético de la zona que se desea prospectar.

\section{MÉTODOS ELECTROMAGNÉTICOS}

Los métodos electromagnéticos reagrupan una gran cantidad de instrumentos diferentes, destinados a medir ciertos parámetros geofísicos como lo son la conductividad eléctrica, la susceptibilidad magnética o la permitividad dieléctrica.

El principio de operación de este método de prospección, está basado en la naturaleza de los campos electromagnéticos que son generados y detectados por bobinas de corriente eléctrica. Si dicho campo logra interactuar con un objeto metálico o cualquier material conductor, sobre éstos se inducen corrientes eléctricas que a su vez producen un campo electromagnético secundario, que es detectado por una bobina de recepción. El valor de la conductividad eléctrica, será medido gracias a la razón de las amplitudes de dichos campos electromagnéticos.

En la Arqueología, se ha intentado emplear este método como sustituto de los métodos eléctricos para evitar de insertar los electrodos en el terreno. En nuestra región, el Complejo Arqueológico de Panamá Viejo [Panamá] y el sitio de Copán - Ruinas [Honduras], han sido objeto de diversas investigaciones geofísicas, y la aplicación de los métodos electromagnéticos a constituido un éxito, no solamente en la detección de restos arqueológicos de tipo hispánico, sino también en el análisis de sus características paleoambientales.

\section{PRESENTACIÓN DE CASOS}

En un marco de cooperación científica entre las universidades francesas (París VI y VII) con los países de la región centroamericana, se han desarrollado diversos trabajos de prospección geofísica aplicados a la detección arqueológica. En este artículo haremos mención a dos sitios importantes: el Complejo Arqueológico de Panamá Viejo y Copán - Ruinas.

\section{Complejo Arqueológico de Panamá Viejo (Panamá)}

Este sitio representa el deseo que surgió en el pasado de crear un punto estratégico, no solamente para la continuidad del proceso de conquista y colonización, sino también como lugar de convergencia para el comercio colonial de la Corona Española. El tráfico de la riqueza proveniente de México, de Perú y Centroamérica hacia España, permitió a la ciudad concentrar una población formada por terratenientes y comerciantes pudientes, y al mismo tiempo convertirse en un foco gubernamental poderoso. $\mathrm{Y}$ es en este marco cultural, que $\mathrm{Pa}$ namá llega a representar el asentamiento español más poderoso e importante de todo el continente americano de la época (Mojica, 2001).

Este conjunto monumental conserva gran parte de sus estructuras, lo que ha facilitado a los arqueólogos una buena delimitación del mismo. Dentro del sitio, se encuentran zonas específicas que contienen características arqueológicas enterradas y estructuras recientes, propias de las actividades del hombre antes de que se declarara patrimonio de la humanidad. Entre las zonas más importantes, podemos señalar:

\section{El Puente del Rey}

Localizado en el sector $\mathrm{N}$ de la parte central del Complejo Arqueológico de Panamá Viejo. El objetivo de la aplicación de las técnicas geofísicas en este sector, se enfocó a detectar parte de una de las calles principales que conectaba el centro de la antigua ciudad con una de las salidas, la cual estaba representada por dicho puente. Esta calle se caracterizaba además porque era la conexión entre el Pacífico y el Atlántico, es decir, que era la vía principal para el transporte de toda la riqueza desde América hacia España. 


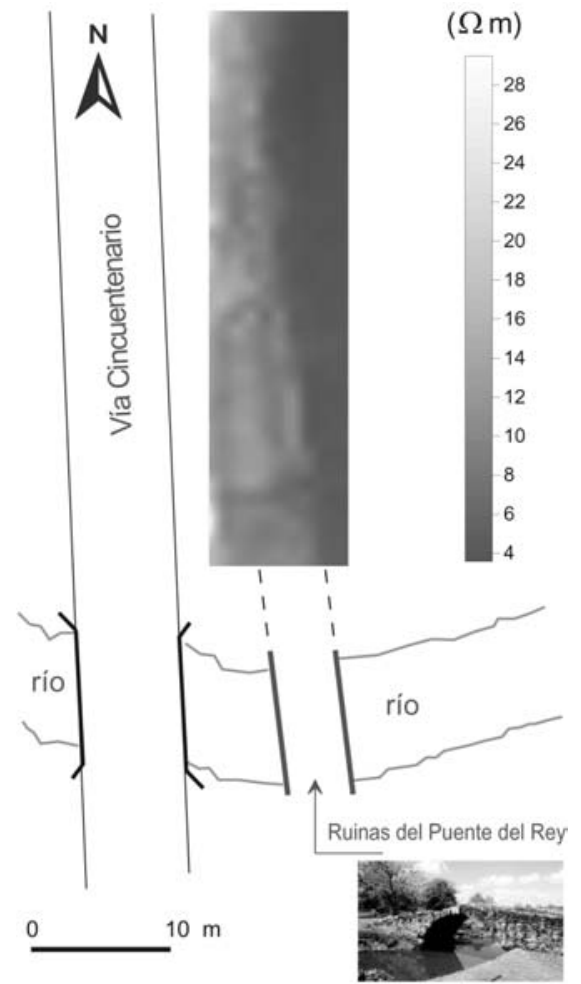

Fig. 1: Mapa de resistividad eléctrica en la zona de Puente del Rey, Panamá.

Se empleó el método de prospección geoeléctrica, en una zona de $400 \mathrm{~m}^{2}$. La frecuencia de medición fue de 1 estación/m para perfiles de $40 \mathrm{~m}$ separados $1 \mathrm{~m}$. Una vez compilada toda la información, los datos fueron sometidos a un tratamiento numérico, el cual permitió revelar la continuación de la calzada (Pastor et al, 2001). La figura 1 muestra los resultados.

La zona rectangular que se encuentra en tonalidades grises, corresponde a la zona prospectada. Cada tonalidad es un indicador de un valor diferente de la resistividad eléctrica del terreno; y es gracias al contraste de este parámetro físico, que se hace posible identificar los límites de la calle que conecta con el puente. Las zonas con valores superiores a $15 \Omega \mathrm{m}$ constituyen la calle, la cual se encuentra a una profundidad que no supera $1 \mathrm{~m}$. La anomalía de alta resistividad eléctrica (en tonalidades claras), con dirección NW - SE, indica los límites de esta ruta hispánica. La línea punteada indica la relación entre dicha anomalía y los restos del puente histórico.

\section{La Casa Alarcón}

Esta estructura hispánica se encuentra hacia el $\mathrm{N}$ del centro del Complejo Arqueológico de Panamá Viejo. El objetivo de la prospección geofísica fue de detectar otras estructuras arqueológicas adyacentes a las ruinas de la Casa Alarcón.

En esta zona, se empleó el método electromagnético Slingram con el equipo EM-38; este sistema posee una separación entre las bobinas de $1 \mathrm{~m}$. Se prospectó un área de $4950 \mathrm{~m}^{2}$, y al igual que la zona descrita anteriormente, la separación entre cada perfil fue de $1 \mathrm{~m}$ y la frecuencia de medición: 1 estación/m.

Gracias a un tratamiento numérico de los datos geofísicos, (aplicación de un algoritmo especial, e interpolación), fue posible identificar algunas anomalías asociadas a ciertas características arqueológicas, y otras, asociadas a material de actividad humana reciente.

En la figura 2 las principales anomalías de la conductividad eléctrica del subsuelo, se han representado por líneas punteadas. En cuanto a

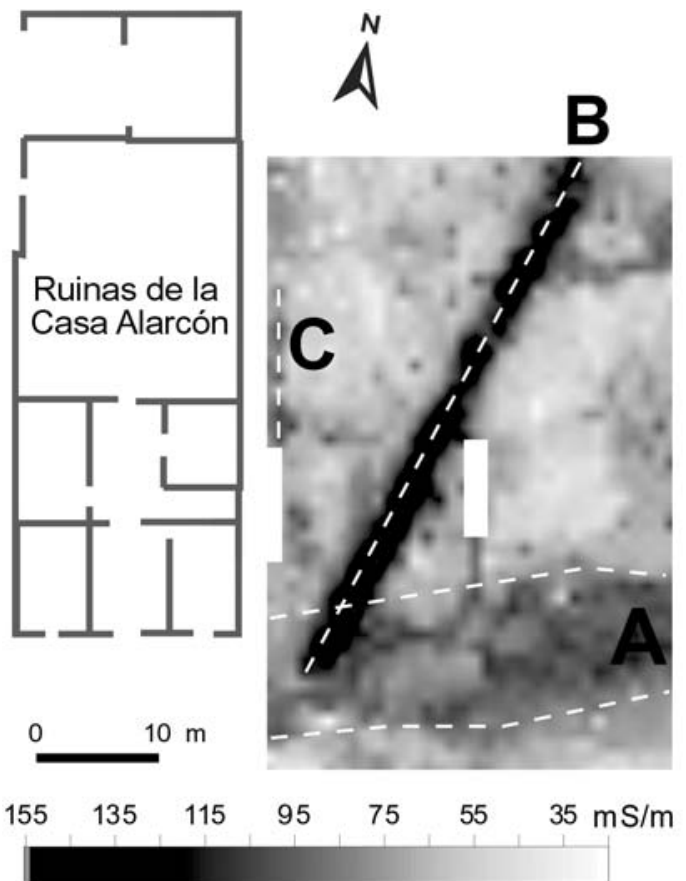

Fig. 2: Mapa de conductividad eléctrica en la zona oriental de las ruinas de la Casa Alarcón, Panamá. 
los contrastes se refiere, las regiones en gris oscuro representan zonas de alta conductividad eléctrica. El pequeño sector en blanco que se encuentra en la parte central, corresponde a una zona no prospectada.

La anomalía A, representada por una zona de conductividad eléctrica intermedia, se encuentra asociadas a una de las vías principales que conectaba el sector central del complejo con las Casas Reales, las cuales se encontraban ubicadas en el extremo oriental del sitio. En estas últimas, se concentraba toda la riqueza, producto de la conquista española y que tendría como destino, España.

La anomalía B, se encuentra asociado a un tubo metálico (reciente). Esta es la anomalía que presenta los más altos valores de la conductividad eléctrica de todo el mapa.

La anomalía $\mathrm{C}$, que contiene valores intermedios de la conductividad eléctrica, se asocia a algunas fundaciones de estructuras de piedra que se encuentran alineadas sobre el terreno.

\section{La zona occidental del sitio}

Esta zona fue estudiada con el gradiente magnético vertical. El objetivo principal de la prospección se enfocó a detectar la continuación de las fundaciones de ciertos muros de tipo hispánico sobre este sector, el cual es utilizado hoy día para eventos sociales.

La superficie prospectada fue de $2021 \mathrm{~m}^{2}$. El equipo utilizado en este análisis corresponde al magnetómetro de protones G-858 de marca Geometrics. Un factor importante que impidió realizar una buena interpretación de los mapas de gradiente magnético, fue la presencia de material férrico disperso, es decir, la contribución de todos estos elementos sobre y alrededor de la zona de estudio (malla metálica, talleres de mecánica y la circulación de automóviles). A causa de este factor, fue necesario llevar a cabo una serie de tratamientos numéricos de datos geofísicos (Pastor et al, 2001).

El mapa de la figura 3 representa el resultado de una sucesión de tratamientos, es decir que los datos del gradiente magnético fueron sometidos a un mecanismo de compresión mediante una función trigonométrica inversa, y sobre dichos valores, se aplicó un filtro paso-bajo (para una ventana de filtraje de $2 \mathrm{~m}$ ) y se interpolaron los valores del gradiente para $(1,0 \mathrm{~m} \times 0,2 \mathrm{~m})$ con el fin de minimizar la contribución negativa de los factores externos.

Las principales características que se pueden identificar, después de haber llevado a cabo un proceso de compresión de valores, de interpolación (definición de una malla de interpolación específica) y filtraje, se especifican a través de las siguientes anomalías del gradiente:

La anomalía A representa la fundación de un muro de tipo hispánico, cuya prolongación parece asociarse a las estructuras de las ruinas localizadas a un costado de la zona de estudio; dicha anomalía posee un alineamiento NE - SW con un elevado grado de magnetización.

La anomalía B puede estar asociada a una construcción reciente. En esta anomalía, los valores altos del gradiente magnético pueden visualizarse de manera puntual, probablemente asociados a elementos metálicos dispuestos verticalmente con un fin de asentamiento (Pastor et al., 2001).

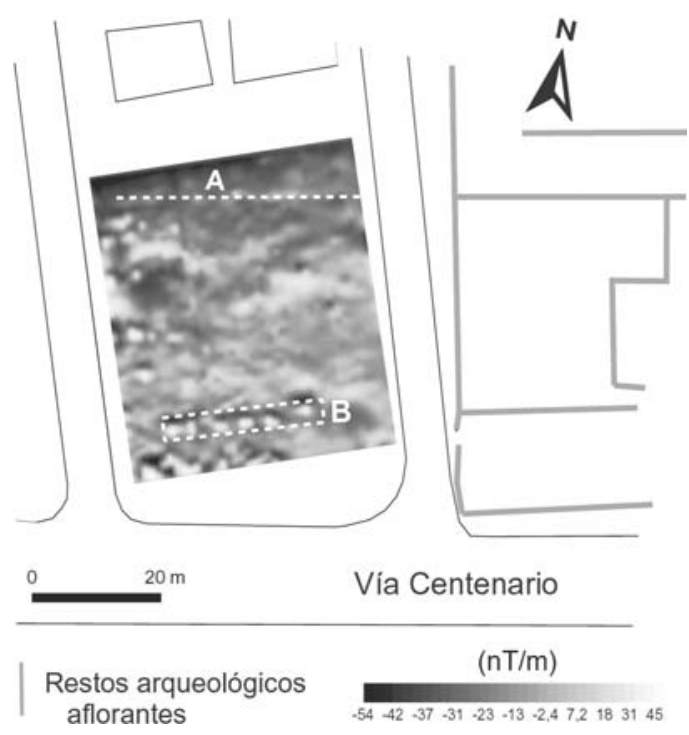

Fig. 3: Mapa del gradiente magnético vertical en la zona occidental del Complejo Arqueológico de Panamá Viejo, Panamá. 


\section{La Acrópolis de Copán (Honduras)}

Localizada en las estribaciones montañosas del oeste de Honduras, la ciudad de Copán ha sido objeto de diversas investigaciones de tipo científica; entre ellas, las prospecciones geofísicas que en los últimos años se han aplicado con el objeto de identificar ciertas estructuras arqueológicas enterradas y reconocer las características paleoambientales del sitio.

La ciudad de Copán correspondió a un importante centro de artesanos. De acuerdo a algunos investigadores (Sedat, 1996 y Murphy, 1999) la consolidación de las creencias y la adoración de los reyes mayas tuvo lugar, pues hoy día es posible observar y estudiar tales características gracias al desarrollo del arte y la escultura.

Uno de los sectores de interés, lo constituyó la zona Oeste del Bosque, sobre la cual se aplicó el método electromagnético Slingram con el equipo EM-31. Este equipo posee una separación de las bobinas de 3,66 m. Se efectuó la medición de la conductividad eléctrica del subsuelo, en donde un total de 874 puntos fueron registrados, en un área 6,30 ha. La frecuencia de medición fue de 1 estación/5 m para perfiles separados una distancia de $5 \mathrm{~m}$.
Los resultados se presentan en la figura 4, donde se identifican dos anomalías importantes: la A es asociada a un meandro antiguo. Los estudios paleoambientales han logrado revelar que se trataba probablemente del antiguo cause del Río Copán. La anomalía B presenta cuatro estructuras bien definidas y se encuentra asociada a las bases de una antigua pirámide.

\section{CONCLUSIONES}

Sin lugar a dudas, la aplicación de los métodos geofísicos, apoyado por el criterio del experto, representa una herramienta de mucha utilidad en el campo de la prospección arqueológica. Es fundamental tener presente las limitaciones de cada uno de los métodos en función de la propiedad física que miden, esto con el fin de poder seleccionar correctamente aquellos métodos que brinden la mejor resolución.

Gracias a la puesta en marcha de estas técnicas modernas, en la región centroamericana hemos logrado identificar la respuesta del efecto del hombre en el pasado, y además, conocer algunas características relacionadas al paleoambiente de algunos de los principales sitios arqueológicos de esta región.

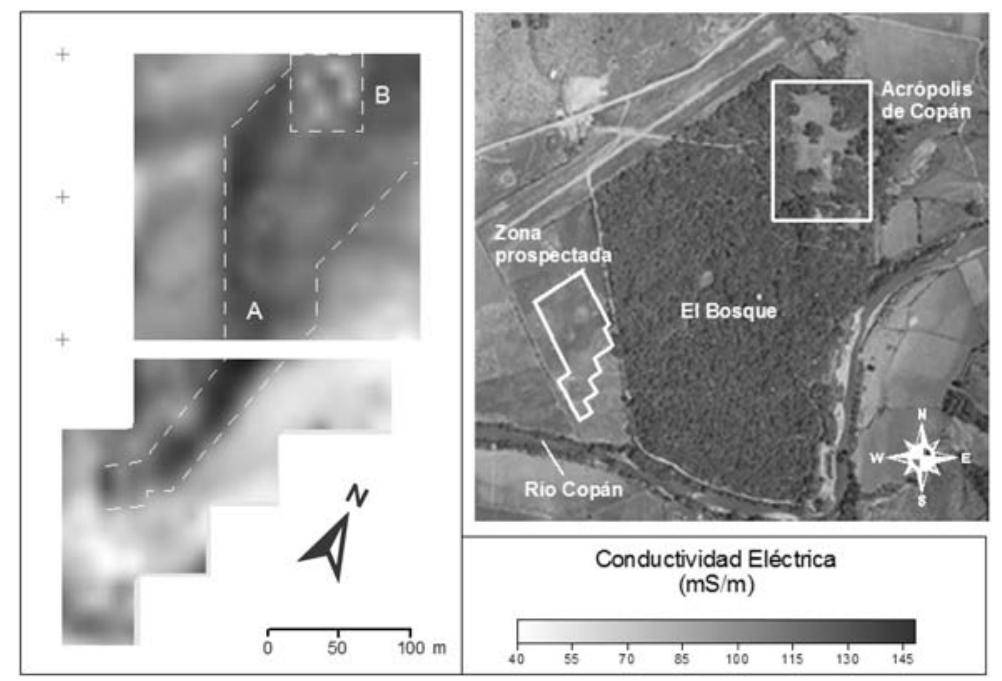

Fig. 4: Fotografía aérea de la zona del Bosque y mapa de conductividad eléctrica del sector oeste; ruinas Copán, Honduras. 


\section{AGRADECIMIENTO}

El aporte brindado por el Centro Cultural y de Cooperación para América Central de la embajada de Francia (CCCAC) en el campo de la geofísica de prospección, y en especial, la aplicada a la Arqueología, es invaluable; ya que ésta ha permitido, no solo la capacitación de personal de las universidades estatales, sino también la realización de importantes estudios en la región centroamericana, contribuyendo de esta manera, al rescate y conservación de nuestro patrimonio cultural.

\section{REFERENCIAS}

ARIAS, M. E., 2000: Métodos geofísicos. - En: DENYER, P. \& KUSSMAUL, S. (comp.): Geología de Costa Rica: 393-409, Ed. Tecnológica de Costa Rica, Cartago.
ARIAS, M. E., 2002: La prospección geoeléctrica y electromagnética en dos dimensiones y su aplicación en la hidrogeología. - Rev. Geól. América Central, 27: 21-26.

DABAS, M., 1989: Comportement magnétique des sols dans le domaine fréquentiel et teporel (VRM). - 213 págs, Univ.París VI, Francia [Tesis de Ph.D.]

MOJICA, A., 2001: Herramienta arqueológica. Periódico La Universidad - I quincena de abril de 2001.

MURPHY, V., 1999: Copán: en el Valle de los Reyes. - Rev. Arqueohispania, 15: 20-32.

PASTOR, L.; VANHOESERLANDE, R.; FLORSCH, N.; FLORSCH, I.; TORAL, J.; GONZALEZ, J.; LEZCANO, M. \& MOJICA, A., 2001: Prospección arqueogeofísica en Panamá La Vieja: Presentación de casos. - Rev. Virtual de Arqueología de Panamá La Vieja: 10-19.

SEDAT, D., 1996: Etapas tempranas en la evolución de la Acrópolis de Copán. - Rev. Yaxkin, 14: 19 - 27. 